\title{
Sistema de apoio a decisão com modelo aditivo para priorização de sistemas de informação
}

\author{
Marina Dantas de Oliveira Duarte (UFPE) marina@ufpe.br \\ Ana Paula Henriques de Gusmão (UFPE) anagusmao@ufpe.br \\ Adiel Teixeira de Almeida (UFPE) aalmeida@ufpe.br
}

\begin{abstract}
Resumo
As organizações estão inseridas em um ambiente dinâmico, tornando cada vez mais relevante a utilização de Sistemas de Informação que as permitam gerir eficazmente seus processos e tomar decisões. Os Sistemas de Informação, dessa forma, exercem grande impacto na competitividade das empresas e, portanto, devem estar alinhados com a estratégia do negócio da organização. A maior dificuldade, todavia, reside na priorização dos módulos de Sistemas de Informação, que deve ser feita em função das limitações de recursos. Esse artigo propõe a utilização de um Sistema de Apoio a Decisão para essa etapa do planejamento de SI, baseado num modelo aditivo.

Palavras chave: Sistema de apoio a decisão, Priorização de Sistemas de Informação, Função aditiva.
\end{abstract}

\section{Introdução}

A decisão de investir em Sistemas de Informaçao (SI) e em Tecnologia de Informação (TI) tem sido alvo, na última década, de inúmeras discussões, pincipalmente pela dificuldade de quantificar os benefícios advindos desse investimento. Entretanto, um ponto neste processo decisório é objeto de consenso na literatura: nenhum investimento em SI será satisfatório e atenderá às expectativas das organizações sem um alinhamento com a estratégia do negócio (ALMEIDA \& COSTA, 2003).

Além da necessidade de transportar a estratégia do negócio para aplicações de SI, as organizações deparam-se com o problema de, depois de identificados os SI mais apropriados e construído o portfólio de projetos de SI, definir a ordem de implementação desses projetos diante da limitação de recursos, o que representa efetivamente a decisão de investimento em SI. Contudo, o cenário atual de mudanças rápidas e freqüentes, onde se encontram as organizações, aumenta o risco de os investimentos de SI falharem (ibidem).

Nesse sentido, esse trabalho apresenta um Sistema de Apoio a Decisão (SAD) para auxiliar a etapa de priorização de Sistemas de Informação, levando em consideração os objetivos organizacionais.

A priorização de Sistemas de Informação está inserida num enfoque maior de Planejamento de SI. Não faz sentido abordar uma metodologia de priorização sem contextualizá-la numa determinada metodologia de Planejamento de SI. Utilizou-se para planejamento de Sistemas de Informação a metodologia Business System Planning (BSP) adaptada.

Na etapa de priorização da BSP, utiliza-se um modelo de decisão multicritério, onde os módulos de Sistemas de Informação são ordenados a partir do estabelecimento de preferências do decisor sobre determinados critérios. Esses critérios abrangem tanto a visão estratégica e processual da organização, quanto os aspectos técnicos. A priorização é, portanto, baseada em agregação aditiva das preferências em relação aos critérios, enfatizando o uso da utilidade multiatributo. Essa etapa é suportada por um software de apoio a decisão, o 
Sistema Multicritério de apoio à decisão para priorização de sistemas de informação com método Aditivo (PSI_A), que consiste no aprimoramento de um sistema anteriormente utilizado, tendo com vantagens, em relação a esse último, a flexibilidade de armazenagem de dados através de um banco de dados e também uma interface mais amigável com o decisor.

\section{Desenvolvimeto de sistemas de apoio a decisão}

Vários autores têm denominado Sistema de Apoio a Decisão a qualquer sistema que forneça alguma contribuição ao processo de tomada de decisões não-estruturadas ou semi-estruturadas (SPRAGUE E WATSON, 1989; BIDGOLI, 1989 \& THIERAUF ,1982).

Um SAD, segundo Sprague \& Watson (1989), caracteriza-se por:

- Direcionar-se, principalmente, para problemas não estruturados, que ocorrem com mais freqüência nos altos níveis gerenciais;

- Combinar a utilização de técnicas de modelagens e analíticas, com funções tradicionais de acesso e recuperação de informações;

- Ter sua interface construída com os usuários visando, principalmente, a facilidade de uso;

- Enfatizar a flexibilidade e adaptabilidade para acompanhamento das mudanças, tanto do ambiente, quanto nas diferentes necessidades de utilização por parte dos usuários.

Segundo Almeida \& Ramos (2002), diferente das outras abordagens de Sistemas de Informação, onde é preciso entender apenas como operar o sistema, num SAD o conhecimento de métodos de apoio a decisão é imprescindível para sua correta utilização. Vários autores sugerem que os decisores atuem de forma integrada com um analista de decisões ou de pesquisa operacional (SPRAGUE \& WATSON, 1989; BIDGOLI, 1989).

Para que o SAD auxilie no processo de tomada de decisão é preciso ser composto de uma base de dados - que auxilia o sistema -, uma base de modelos - que provê a capacidade de análise - e o diálogo - que provê a interação entre o usuário e o sistema. A relação entre esses elementos constitui a arquitetura de um SAD e pode ser visualizada na figura a seguir.

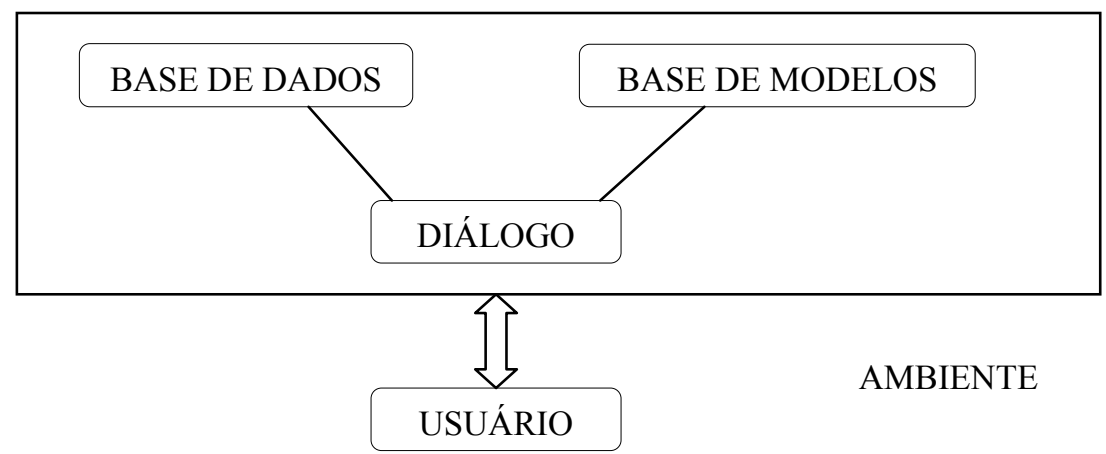

Figura 1 - Arquitetura de um SAD (adaptada de Bidgoli, 1989)

$\mathrm{Na}$ base de dados, estão presentes os dados internos e os externos, que podem se acessados diretamente pelo usuário ou entram como input para a base de modelos. Os dados internos referem-se aos conhecimento a priori de gerentes e aos dados provenientes dos sistemas transacionais da organização. Já os dados externos são necessários especialmente quando as decisões são dos níveis mais altos da organização. De acordo com Gomes et al. (2002), a base de dados deverá fornecer as informações de forma rápida e permitir sua manipulacão de forma eficiente. 
A base de modelos provê a capacidade de análise para o SAD. Usando uma representação matemática do problema, os algoritmos servem para gerar informação para auxiliar a tomada de decisão (ALMEIDA \& RAMOS, 2002).

O diálogo pode ser entendido como uma combinação de software, hardware e pessoas, que permite uma interação entre o usuário e o SAD. Trata-se do componente mais importante para o usuário, pois para ele o diálogo é a forma como o sistema opera e funciona.

No desenvolvimento de um SAD, deve-se considerar inicialmente três tecnologias básicas de sistema de apoio a decisão, as quais devem ser entendidas pelos desenhistas e pelos usuários. São elas SAD específico, Gerador de SAD e Ferramentas SAD. O SAD específico é uma combinação de hardware e software usada para apoiar o tomador de decisão em uma tarefa específica. O gerador de SAD usa uma combinação de hardware e software como um pacote para desenvolver um SAD específico. Já as Ferramentas SAD são usadas para desenvolver ou um SAD específico ou um Gerador de SAD (ALMEIDA \& RAMOS, 2002).

Sprague \& Watson (1989) destacam com muita ênfase, no desenvolvimento de um SAD, a importância de uma abordagem evolutiva, de modo que o sistema possa partir de uma versão menor do problema evoluir até uma versão mais abrangente. A idéia para começar é reduzir o tamanho do problema para que o SAD possa atender de início a uma parte do problema e assim ter o seu desenvolvimento evolutivo à medida que a equipe aprende mais sobre a natureza do problema não estruturado.

Baseado nessa abordagem evolutiva dos Sistemas de Apoio a Decisão, foi idealizado o software PSI_A, que auxilia a etapa de priorização de Sistemas de Informação, e que constitui numa evolução de um sistema já desenvolvido com esse objetivo. O PSI_A, todavia, apresenta um desempenho mais eficaz em relação ao suporte do processo de tomada de decisão.

Na seção a seguir, será apresentada a etapa de priorização, bem como o modelo multicritério de apoio a decisão utilizado para suportá-la.

\section{Modelo de decisão multicritério para a etapa de priorização de SI}

A metodologia Business System Planning foi lançada pela Internacional Business Machines Corporation (IBM) em meados da década de setenta. O fundamento da BSP é de que a informação é um recurso a ser utilizado por toda a organização e, por conseguinte, deve ser planejada sob este enfoque.

Utilizou-se nesta pesquisa uma adaptação da metodologia BSP, tendo em vista simplificações de alguns estágios e estruturação de vários aspectos observados na priorização. Esta metodologia consiste em duas etapas principais: a elaboração de um estudo para se obter a visão estratégica da organização, sintetizada na forma de fatores estratégicos, e a Engenharia da Informação, que ressalta a construção da arquitetura de Sistemas de Informação da organização.

A arquitetura do Sistema de Informação contempla os Agrupamentos de Informações (AGI) que representam o relacionamento entre os processos da organização e as classes de dados, e os diversos Tipos de Tecnologias de Informação (TSI). A combinação destas duas visões forma os Módulos de Serviço de Informação (MSI), estes representam as aplicações ou projetos de sistemas de informação. Esta fase da metodologia é composta por três etapas: Dados Corporativos, Modularização e Priorização. As aplicações de SI são priorizadas tendo como objetivo o suporte à consecução dos resultados esperados da organização (COSTA et al., 2002). 
$\mathrm{Na}$ etapa de Priorização, no entanto, a metodologia não apresenta um suporte mais formal, apenas procedimentos ad hoc foram aplicados na maioria dos casos. Dessa forma, para suportar esta etapa de modo mais estruturado, foram desenvolvidas algumas abordagens tratando o problema de priorização de Sistemas de Informação como um problema de apoio a decisão multicritério, pois de fato o que se deseja é, em função de uma série de critérios, ordenar todas as aplicações, classificá-las em grupos, para finalmente escolher um ou mais grupos para implementar num primeiro horizonte.

Modelos de decisão foram construídos, todos baseados em métodos de apoio a decisão multicritério, que abordam de forma diferente o problema de priorização (Almeida \& Ramos, 2002). Neste artigo, o modelo de decisão baseia-se num procedimento de agregação dos critérios analisados, na forma de uma função valor aditivo linear (ALMEIDA, 1999).

Essa etapa, todavia, não deve ser realizada levando em consideração apenas aspectos financeiros, mas principalmente os objetivos empresariais. O processo de Priorização consiste em construir uma matriz de priorização que apresente pesos, para cada MSI, obtidos através da análise relativa de fatores relevantes entre os AGI's e entre os TSI's que os compõem.

As informações de natureza técnica são fornecidas pelos especialistas em Sistemas de Informação que participaram da etapa de engenharia de informação. As informações de natureza decisória são obtidas diretamente do decisor.

O decisor estabelece os pesos relativos para a dimensão AGI e a dimensão TSI e a avaliação de cada MSI é obtida através do somatório das duas visões de ponderação ponderadas pelos pesos atribuídos às dimensões AGI e TSI, obtém-se então o ordenamento inicial das aplicações de SI (COSTA et al., 2002).

A última etapa da BPS adaptada consiste no Plano de Ação que define os projetos a serem desenvolvidos, partindo-se das prioridades determinadas e da análise da estrutura de hardware, software e recursos humanos envolvidos.

\section{O software PSI_A}

$\mathrm{O} \mathrm{SAD}$, por ser um sistema orientado a problemas não-estruturados ou semi-estruturados, apresenta uma metodologia de desenvolvimento baseada na abordagem evolutiva. De acordo com essa abordagem, os Sistemas de Apoio a Decisão devem estar sempre evoluindo, visando agregar novos recursos, de forma a suportar de forma mais eficaz o processo decisório.

Dessa forma, o Sistema de Apoio a Decisão PSI_A foi construído a partir de outro concebido anteriormente também com o objetivo de aplicar o modelo descrito na seção anterior e suportar a etapa de priorização. Com esse novo sistema, busca-se prover mais recursos para a execução processo decisório, armazenar dados de processos de priorização e apresentar uma interface mais amigável em relação ao sistema anteriormente utilizado.

No SAD desenvolvido nesse trabalho, a base de dados é constituída por informações obtidas diretamente do decisor e do especialista em SI e pelos resultados obtidos da metodologia de planejamento de SI.

O elemento base de modelos é construído através de uma série de elementos e suas relações. O modelo do SAD PSI_A baseia-se na modelagem de preferências do decisor, combinando os vários critérios considerados numa função valor aditivo, apresentando assim um tipo de compensação entre os critérios. Intuitivamente esta noção sugere uma quantidade que compense a desvantagem de um critério em relação a uma vantagem em outro.

No SAD desenvolvido, optou-se por um uma certa flexibilidade, enfatizando o diálogo direto. Ele foi desenhado para o uso interativo, o usuário e o diálogo interagem diretamente, embora 
seja permitida a existência ou não de uma terceira parte envolvida no processo. Segundo Costa et al. (2002), é possível considerar a existência de um analista de apoio. O analista é capaz não só de operar o sistema como também de interpretar seus resultados. Na verdade ele assiste o tomador de decisão que não está familiarizado com os diferentes tipos de analises realizadas pelo SAD (BIDGOLI, 1989).

O PSI_A estabelece uma relação entre base de modelos e base de dados, que acontece da seguinte forma: o modelo recebe dados da base de dados, estes dados são o resultado da aplicação da metodologia de planejamento e dados obtidos diretamente do decisor e especialistas em SI, gerando como recomendação para o decisor as aplicações prioritárias de SI.

Seu algoritmo segue os passos do modelo, sendo inicialmente requerida a entrada dos AGI's, processos, fatores estratégicos, critérios de processo, TSI's e fatores de ponderação dos TSI's, que serão utilizados pelo decisor em seguida (COSTA et al., 2002).

Na Figura 2, pode-se observar a solicitação, por parte do sistema, dos processos que farão parte da priorização.

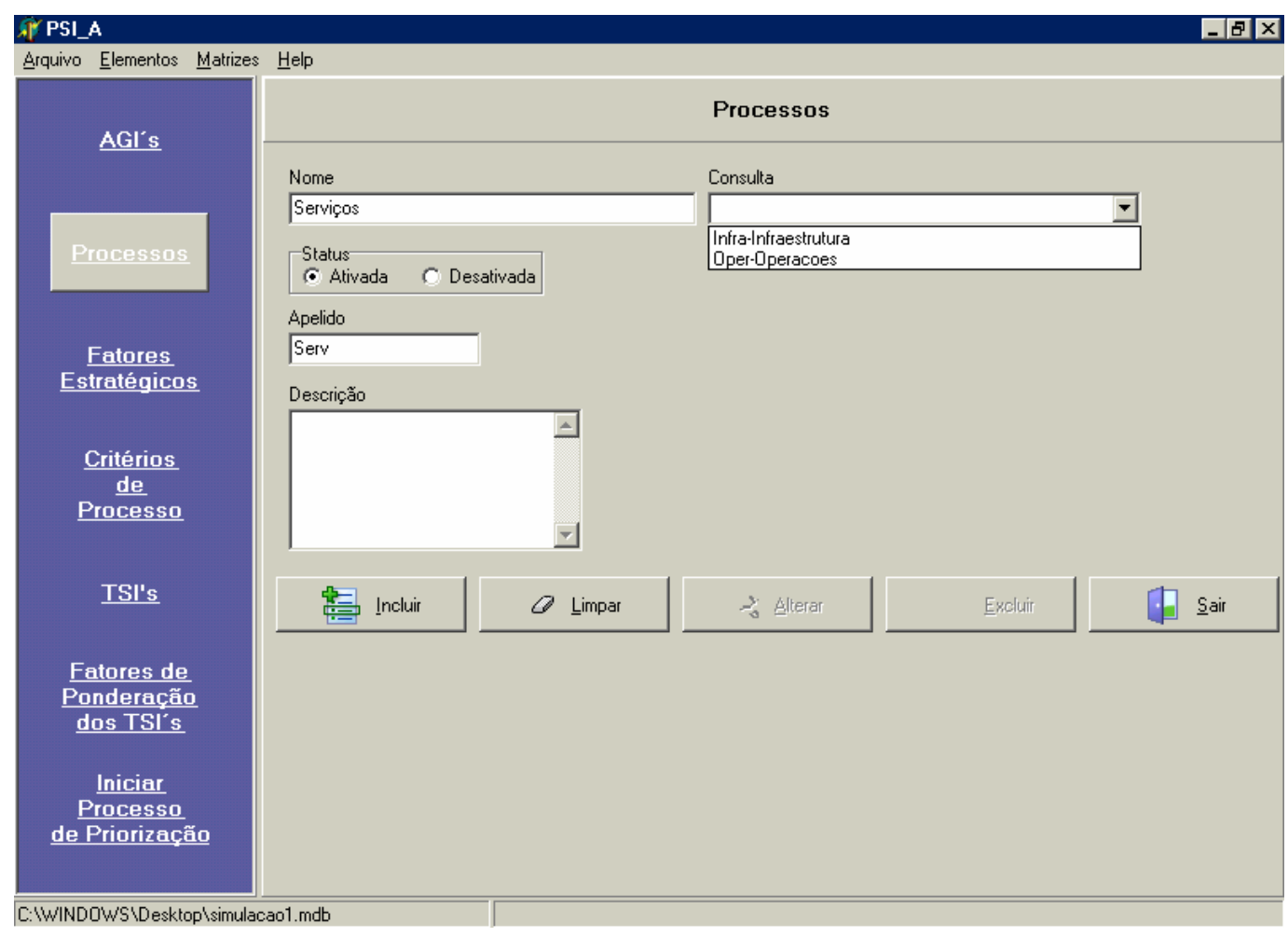

Figura 2 - Exemplo de entrada de dados

Em seguida, o sistema gera as matrizes das relações dos dados de entrada, as quais devem ser preenchidas com os valores atribuídos pelo decisor, como é exemplificado pela Figura 3. 


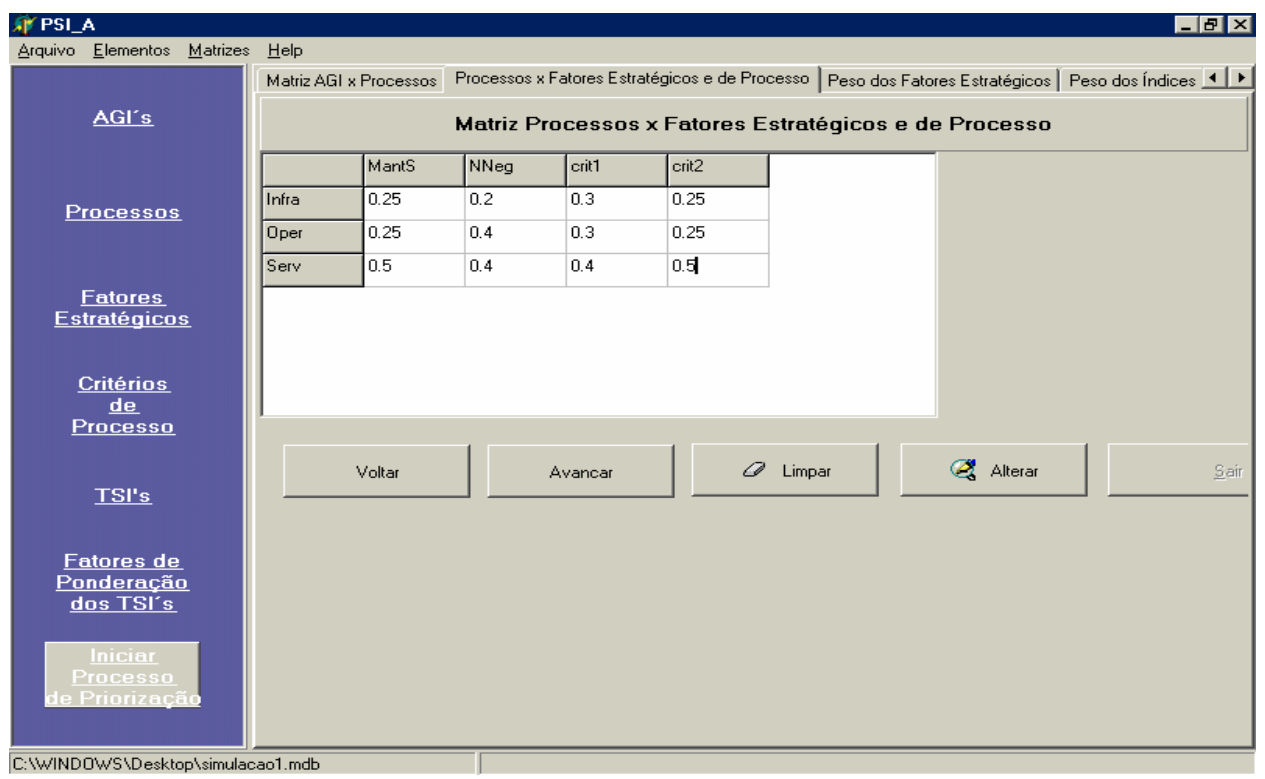

Figura 3 - Exemplo de matriz das relações

De posse destas relações, o programa fornece a matriz final de avaliação dos MSI's, a qual é exemplificada na Figura 4. A ordenação dos valores desta matriz final fornece uma relação dos módulos de sistemas de informação classificados por importância. É necessário ressaltar que, em função dos procedimentos utilizados, esta relação é ordinal, ou seja, não se deve considerar a razão entre os pesos obtidos como indicativo de importância relativa, não há cardinalidade (ALMEIDA \& RAMOS, 2002).

O decisor, para análises mais detalhadas, pode requerer também do sistema a visualização das matrizes intermediárias descritas pelo modelo.

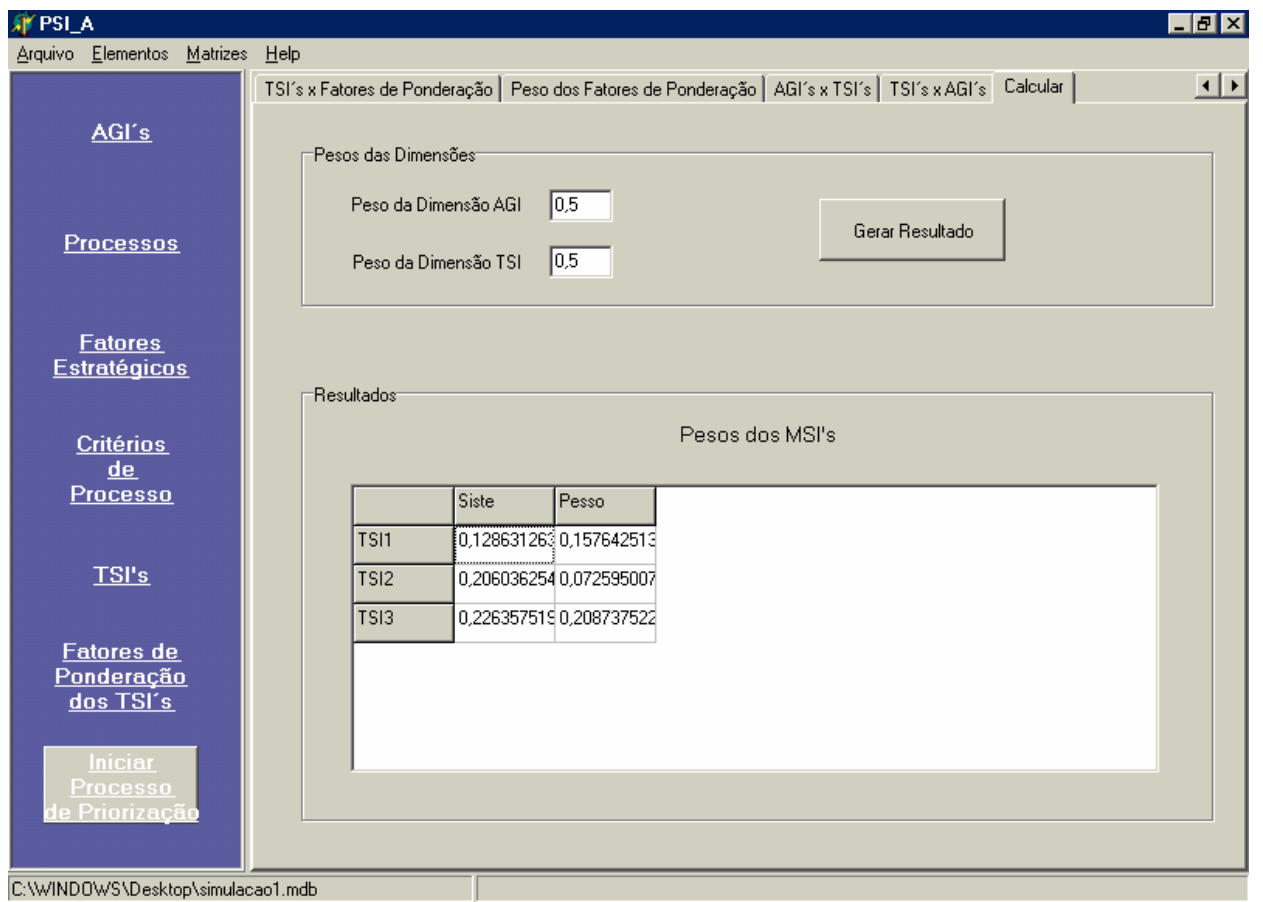

Figura 4 - Exemplo de avaliação dos MSI’s

\section{Conclusões}

A informação constitui-se, atualmente, em um bem estratégico para as organizações, que 
buscam se manter competitivas. As empresas precisam apresentar grande capacidade de flexibilidade e adaptação às mudanças constantes do presente ambiente, altamente dinâmico. É necessário, no entanto, um controle sistemático de gerenciamento para que a informação, alinhada aos objetivos organizacionais, venha a desempenhar um papel estratégico dentro das empresas. Assim, são desenvolvidos os Sistemas de Informação.

Para que os SI venham desempenhar seu papel de forma eficaz dentro das empresas é necessário que haja um planejamento destes sistemas. Esse planejamento, dentre outras coias, visa alinhar os objetivos dos SI com os objetivos estratégicos da organização como um todo.

Dentro desse contexto, foi desenvolvido um modelo para suportar a etapa de priorização do processo de Planejamento dos Sistemas de Informação, que se baseia numa agregação aditiva das preferências do decisor em relação aos critérios. Dessa forma, a etapa de priorização é tratada como um problema de apoio a decisão multicritério. Em função de uma série de critérios, todas as alternativas serão ordenadas, classificadas em grupos e finalmente serão escolhidos um ou mais grupos para serem implementados em curto prazo. O modelo de decisão multicritério utilizado baseia-se na função utilidade aditiva linear.

Com o objetivo de simplificar a execução do processo de priorização dos Sistemas de Informação, foi construído o Sistema de Apoio a Decisão PSI_A, que constitui num aprimoramento de outro sistema desenvolvido anteriormente. Esse sistema interage com o decisor, obtendo dele os dados necessários a realização dos cálculos, que serão exibidos em forma de matrizes.

\section{Referências}

ALMEIDA, A.T. de (1999a) - Um Modelo de Decisão para Priorização no Planejamento de Sistemas de Informação. Revista Produção. Vol. 8, n 2, p. 169-185.

ALMEIDA, A.T. \& RAMOS, F.S. (2002) - Gestão da Informação na Competitividade das Organizações. Editora Universitária da UFPE. Recife.

ALMEIDA, A.T. \& COSTA, A.P.C.S. (2003) - Aplicações com Métodos Multicritério de Apoio a Decisão. Editora Universitária da UFPE. Recife.

BIDGOLI, H. (1989) - Decision Support Systems - Principles and Practice. West Publishing Company. New York.

COSTA, A.P.C.S; GUSMÃO, A.P.H. \& ALMEIDA, A.T. (2002) - Sistema de Apoio a Decisão para Priorização de Sistemas de Informação. In: Encontro Nacional de engenharia de Produção, 22. Curitiba. Anais...Curitiba: Abepro, 2002. CD-ROM.

GOMES, L.F.A.M.; GOMES, C.F.S. \& ALMEIDA, A.T. (2002) - Tomada de Decisão Gerencial: Enfoque Multicritério. Editora Atlas. São Paulo.

SPRAGUE Jr, R.H.; WATSON, H.J. (1989) - Decision Support Systems - Putting Theory into Practice, Prentice-Hall, Inc.

THIERAUF, R.J. (1982) - Decision support systems for effective planning and control - A case study approach. Prentice-Hall, Inc. New Jersey. 